УДК 338.242.4

DOI 10.17308/econ.2020.1/2763

\title{
ЦИФРОВЫЕ ПЛАТФОРМЫ В ПУБЛИЧНОМ УПРАВЛЕНИИ НА ОСНОВЕ AGILE МЕНЕДЖМЕНTA
}

\author{
А. В. Полянин, И. А. Докукина \\ Среднерусский институт управления - филиал РАНХиГС
}

Поступила в редакцию 14 ноября 2019 г.

\begin{abstract}
Аннотация: представлена и обоснована авторская перекрестная классификация цифровых платформ в зависимости от того, кто владеет активом и устанавливает ичены и другие условия, а такюе в зависимости от типологии программного продукта и возможности обмена цеенностями. Детализированы девять типов ичифровых платформ и дана их развернутая характеристика. Составленная авторами классификация позволяет применить различные типь ичифровых платформ к различным уровням публичного управления. На всех уровнях публичного управления, руководствуясь концепциями Government as a Platform (GaaP) и Government 2.0, авторы обосновывают применение циифровых платформ для координации инновационных моделей, предоставления публичных услуг и внутреннего взаимодействия между уровняли публичного управления и внутри них (адаптеры). Постоянное изменение внешней и внутренней среды требует обеспечивать гибкость в применении циифровых платформ, постоянной их модернизации и улучшении качественных характеристик. Поясняется, почему применение иченностей и принципов agile менеджмента необходимо для создания и использования циифровых платформ в публичном управлении. В зависимости от типовых характеристик уровня публичного управления предложены наиболее цеелесообразные для применения типы ццифровых платформ на данном уровне.
\end{abstract}

Ключевые слова: иүифровая платформа, публичное управление, agile менеджмент, теория сложности, теория сетей.

Annotation: the article presents and justifies the author's cross-classification of digital platforms depending on who owns the asset and sets prices and other conditions, as well as depending on the typology of the software product and the ability to exchange values. Nine types of digital platforms are detailed and their detailed characteristics are given. The classification compiled by the authors makes it possible to apply different types of digital platforms to different levels of public administration. At all levels of public administration, guided by the concepts of Government as a Platform (GaaP) and Government 2.0, the use of digital platforms for coordinating innovative models of public service delivery and internal interaction between and within the levels of public administration (adapters) is justified. Constant changes in the external and internal environment require flexibility in the use of digital platforms, their constant modernization and improvement of quality characteristics. The article explains why the application of agile management values and principles is necessary for the creation and use of digital platforms in public management. Depending on the typical characteristics of the level of public management, we have proposed the most appropriate types of digital platforms for use at this level.

Key words: digital platform, public management, agile management, complexity theory, network theory.

При взаимодействии в публичном управлении, прежде всего между его уровнями: государственным, региональным, муниципальным и негосударственным, необходимо учитывать законы сложности и понимать, что современное управление не может строиться только на принципах детерминизма и причинно-следственных связях. Детерминизм основывается на постулате, что причиной любого события является другое событие, ранее произошедшее. Хотя в мире науке применение детерми-

(c) Полянин А. В., Докукина И. А., 2020 низма позволило сделать множество открытий, но в современных системах управления его недостаточно. На наш взгляд, невозможно предвидеть точно результат сложной конфигурации желаемых и фактических параметров. Как точно выразился Г. Л. Менкен: «Всякая сложная проблема имеет решение - простое, удобное и ошибочное» [1].

Ошибочные решения в управлении возникают прежде всего из-за того, что мозг человека предпочитает видеть простые причинно-следственные связи и избегать сложности. Майкл Брукс в своей статье «Рожденные верить: Как наш мозг создает 
богов» отмечает, что «человеческий мозг чрезмерно ориентирован на установление причинно-следственных связей, что заставляет нас видеть их даже там, где их нет» [2]. Наш мозг эволюционно построен отдавать предпочтение линейному мышлению. На самом деле большинство процессов основываются на нелинейных событиях. Деятельность нашего мозга, построенная на линейном мышлении, воспринимает мир как пространство, наполненное причинно-следственными связями. Джеральд Вайберг называет такой подход в управлении системной ошибкой причинности [3].

Если развитие науки с XVII в. проходило под знаком детерминизма, то со средины XX в. предметом исследований стали сложные системы. На современно этапе такие научные направления, как теория динамических систем, теория хаоса, теория игр, теория сетей и ряд других, можно объединить собирательно теорией сложности. Развитие теории сложности позволяет применить новый научный подход к проблемам управления организациями.

Одна из важнейших, на наш взгляд, концепций теории сложности по отношению к системам управления заключается в том, что все организации представляют собой сети. Мы можем представлять организации в виде иерархий, различных организационных структур (линейных, дивизионных, матричных и т. д.), но на практике они все будут сетями. Теория сложности по отношению к социально-экономическим системам показывает, что менеджмент в первую очередь должен заниматься людьми и их взаимоотношениями, а не организационными структурами и получением экономического эффекта. Не отвергая необходимые и полезные инструменты, созданные в научных разработках, применяемых в иерархическом менеджменте, на современном этапе в основных положениях по управлению организациями необходимо придерживаться принципов и научных положений, связанных с сетевыми структурами.

Когда иерархический менеджмент сталкивается со сложными системами и нелинейным мышлением, необходимо переводить управление организациями в плоскость гибкого (agile) менеджмента. Согласимся с мнением Ю. И. Трещевского: «Неопределенность является объективной характеристикой соответствующей ситуации» [4], и отметим, что именно неопределенность является главной характеристикой сложной системы. Социально-экономические системы, в которых осуществляют свою деятельность органы публичного управления, являются сложными системами, и именно исходя из постулатов теории сложности к ним в первую очередь необходимо применять принципы agile менеджмента.

Фундаментальные принципы agile менеджмента были неоднократно описаны многими авторами, мы же предлагаем раскрыть противопоставление ценностей в иерархическом и agile менеджменте (табл. 1). Органы публичного управления должны перестроить свою работу, не исключая применение основополагающих принципов и методов иерархического менеджмента, им необходимо внедрять ценности agile менеджмента. Причем в деятельности необходимо: 1) ценить людей и взаимодействие между ними больше, чем процессы и инструменты; 2) ценить работающий продукт (услугу) больше, чем полную документацию; 3 ) ценить сотрудничество с заказчиком больше, чем условия первоначального контракта; 4) ценить готовность реагировать на изменения больше, чем четкое выполнение первоначального плана.

Методологической основой сочетания основных принципов и функций иерархического менеджмента с ценностями agile менеджмента можно предложить системное мышление, которое фокусируется на циклических взаимоотношениях между компонентами системы и нелинейных связях внутри нее. Такое сочетание позволяет избежать непредвиденных последствий, риск возникновения которых повышается, если компоненты управления рассматриваются изолированно. Основная ценность системного мышления состоит в том, что оно позволяет объектам и субъектам управления рассмотреть проблему с различных ракурсов [5].

Если теоретической основой мы определяем системное мышление, то практическим адаптером,

Т а б ли ц а 1

Противопоставление иенностей в иерархическом и agile менеджменте

\begin{tabular}{|c|c|c|}
\hline Ценности agile менеджмента & & Ценности иерархического менеджмента \\
\hline Люди и их взаимодействие & $\geq$ & Процессы и инструменты управления \\
\hline Работающий продукт & $\geq$ & Полная документация \\
\hline Сотрудничество с заказчиком & $\geq$ & Условия контракта \\
\hline Реагирование на изменения & $\geq$ & Четкое выполнение плана \\
\hline
\end{tabular}


обеспечивающим симбиоз иерархического и agile менеджмента, в современных условиях, на наш взгляд, являются цифровые платформы. Цифровая платформа, как система алгоритмизированных взаимовыгодных взаимоотношений большого количества участников процесса (сферы деятельности), позволяет в единой информационной среде обеспечивать взаимовыгодность отношений объектов и субъектов публичного управления. Причем выгода может иметь не только экономический характер, но и социальную направленность. Наиболее важно для органов публичного управления то, что взаимодействие участников с помощью цифровой платформы может обеспечивать социальный эффект. Этот эффект достигается за счет применения определенных технологий и модернизации (реструктуризации) отдельных бизнес-процессов. В современных условиях, когда все организационно-управленческие механизмы внутри организации и при ее взаимодействии с внешней средой строятся по сетевому принципу, именно цифровые платформы помогают участникам социально-экономических процессов осуществлять эффективное взаимодействие, добиваясь экономической выгоды или социального эффекта.

В современной научной литературе цифровые платформы рассматриваются и классифицируются в зависимости от исследовательского интереса и цели исследования, различные научные школы предлагают различные классификации и их научные обоснования [6]. В настоящее время есть множество классификаций в рамках развития таких направлений экономической науки, как неоинституциализм, теория информационных процессов, теория инновационного менеджмента, теория сложности (в подразделах теории динамических систем, теории хаоса, теории игр, теории сетей) и др. Наиболее часто схематично цифровые платформы подразделяют по формату [7], т. е. направлению их использования: социальные сети, мессенджеры, поисковые системы, видеоплатформы, интернет-браузеры, операционные системы, платежные системы, краундфандинг, поиск работы, электронная торговля, пассажирские перевозки, аренда жилья, туристические услуги, государственные услуги и т. д.

На наш взгляд, цифровые платформы с точки зрения их внедрения в систему публичного управления наиболее целесообразно классифицировать:

а) в зависимости от того, кто владеет активом и устанавливает цены и другие условия: централизованные, децентрализованные, гибридные; б) в зависимости от типологии программного продукта и возможности обмена ценностями: инфраструктурные, инструментальные, прикладные.

Наше авторское предложение заключается в том, что необходимо не только классифицировать цифровые платформы по вышеуказанным признакам, но и перекрестно объединить их с целью установления сущностных характеристик и уникальных особенностей для общества и государства. В табл. 2 представлены примеры перекрестной классификации в зависимости от владения правом на установления условий (владения активом) и типологии программного продукта.

Инструментальные цифровые платформы предназначены для программных решений на основе сквозных технологий работы с данными, в инструментарий уже включены типовые функции и интерфейсы, которые позволяют осуществлять программно-аппаратные решения. Основной вид деятельности на базе платформы - программно-аппаратные решения. Результатом деятельности на цифровой платформе будет программное средство (продукт) для обработки информации и технологические операции обработки информации. Основным бенефициаром станет разработчик программных решений.

В то же время инструментальные цифровые платформы будут подразделяться на различные типы в зависимости от того, кто владеет активом и устанавливает цены и другие условия. Если рассматривать централизованные инструментальные цифровые платформы (тип А), то владелец актива устанавливает практически все условия, в том числе масштабирование и цены, осуществляет контроль за качеством и стандартизацией. Для создания такой платформы необходим значительный авансированный капитал, первоначальные затраты будут самые высокие по сравнению с созданием других платформ. Типичными примерами цифровых платформ типа А являются поисковые системы (Google; Bing; Yahoo; Baidu; Mail; Yandex; Sputnik; Rambler) и операционные системы для персональных компьютеров (Linex; MacOs; MAKC; Windows; RAIDIX; KasperskyOS). Главное преимущество таких платформ - документированные интерфейсы доступа к данным, также следует отметить, что себестоимость снижается путем многократного использования инструментария работы с данными.

В децентрализованных инструментальных цифровых платформах (тип D) владелец, устанавливая условия, предлагает актив непосредственно 
Авторская перекрестная классификация цฺифровых платформ (ЦП) с примерами

\begin{tabular}{|c|c|c|c|}
\hline $\begin{array}{l}\text { ЦП по владению } \\
\text { активом и установлению типологии } \\
\text { условий }\end{array}$ & $\begin{array}{c}\text { Инструментальная } \\
\text { ЦП - в основе находит- } \\
\text { ся программно-аппа- } \\
\text { ратный комплекс }\end{array}$ & $\begin{array}{c}\text { Инфраструктурная ЦП - } \\
\text { в основе экосистема } \\
\text { участников по автомати- } \\
\text { зации их деятельности }\end{array}$ & $\begin{array}{c}\text { Прикладная ЦП - } \\
\text { в основе находится } \\
\text { бизнес-модель по } \\
\text { ведению хозяйственной } \\
\text { деятельности }\end{array}$ \\
\hline $\begin{array}{l}\text { Централизованная ЦП - владеет } \\
\text { активом и устанавливает условия, } \\
\text { обеспечивает стандартизацию и } \\
\text { масштабирование }\end{array}$ & \begin{tabular}{l}
\multicolumn{1}{c}{ A } \\
примеры: Google; Bing; \\
Yahoo; Baidu; Mail; Java; \\
Yandex; Rambler; MacOs; \\
Sputnik; Windows; Linex; \\
KasperskyOS; Bitrix
\end{tabular} & \begin{tabular}{l}
\multicolumn{1}{c}{ B } \\
примеры: WhatsApp; Tele- \\
gram; WeChat; Tamtam; \\
Apple iOS; Skape; Avirton; \\
Farechat; Android; Sail- \\
fishOS; WindowsPhone
\end{tabular} & \begin{tabular}{l}
\multicolumn{1}{c}{ C } \\
примеры: Zipcar; T \\
visor; Rent the runu \\
Aviasales; Tourviso \\
Travelata; Skyscann \\
Kayak; Gosuslugi
\end{tabular} \\
\hline $\begin{array}{l}\text { Децентрализованная ЦП - вла- } \\
\text { делец устанавливает условия и } \\
\text { предлагает актив непосредствен- } \\
\text { но пользователю }\end{array}$ & $\begin{array}{l}\text { D } \\
\text { примеры: Facebook; } \\
\text { Twitter; Instagram; } \\
\text { Linkedin; Chrome; Vk; } \\
\text { Odnoklassniki; Firefox; } \\
\text { Opera; Safari }\end{array}$ & $\begin{array}{l}\mathrm{E} \\
\text { примеры: AirBnB; Ho- } \\
\text { meAway; Cian; Hostel- } \\
\text { world; Udacity; Domo- } \\
\text { fond; Coursera; Stepik; } \\
\text { Universarium; Edx }\end{array}$ & \begin{tabular}{l}
\multicolumn{1}{c}{ F } \\
примеры: Groupon; \\
Kickstarter; Planeta; \\
IndieGoGo; Starttrack; \\
Boomstarter; Biglion; \\
Crowdfunder
\end{tabular} \\
\hline $\begin{array}{l}\text { Гибридная ЦП - владение и риск } \\
\text { децентрализованы, а стандарти- } \\
\text { зация и уровень обслуживания } \\
\text { централизованы }\end{array}$ & \begin{tabular}{l}
\multicolumn{1}{c}{ G } \\
примеры: Youtube; Vi- \\
meo; MetaCafe; Rutube; \\
Ozon; Ivi; Aliexpress; \\
Amazon; Ticketland; Etsy
\end{tabular} & \begin{tabular}{|l}
\multicolumn{1}{|c|}{ H } \\
примеры: WePay; PayPal; \\
Amazon Pay; Apple Pay; \\
Robokassa; QIWI; eBay
\end{tabular} & $\begin{array}{l}\text { I } \\
\text { примеры: Lyft; Uber; } \\
\text { Blablacar; City-mobil; } \\
\text { Indeed; Job; Upwork; } \\
\text { Rentmania; CareerBuilder }\end{array}$ \\
\hline
\end{tabular}

пользователю. Роль цифровой платформы в том, что она позволяет осуществлять коммуникационное взаимодействие агентам и облегчает трансакции. Преимуществом при создании являются низкие первоначальные затраты, но в то же время платформа должна постоянно привлекать агентов для обеспечения нормализованного предложения. Типичными примерами цифровых платформ типа D являются социальные сети (Facebook; Twitter; Instagram; Linkedin; Vk; Odnoklassniki) и интернет-браузеры (Firefox; Opera; Chrome; Safari; Microsoft Edge).

В гибридных инструментальных цифровых платформах владелец актива предлагает услугу с ценой и стандартами. Владение и риск в данном случае децентрализованы, а стандартизация и уровень обслуживания централизованы. Примерами гибридных инструментальных цифровых платформaх типа G являются видеоплатформы (Youtube; Vimeo; MetaCafe; Rutube; Ivi) и платформы электронной торговли (Ozon; Aliexpress; Amazon; Ticketland; Etsy). Как и в децентрализованных инструментальных цифровых платформах, первоначальные капитальные затраты низкие, но привлечение агентов имеет решающее значение.

Основной вид деятельности инфраструктурных цифровых платформ - предоставление ИТ-сервисов и информации для принятия решений. Результатом деятельности на инфраструктурной цифровой плат- форме является информация, необходимая для принятия решений на основе результатов работы ИТ-сервиса. Основным бенефициаром будет продуктолог (заказчик ИТ-сервиса для потребителя). Инфраструктурная цифровая платформа имеет определенные группы участников: разработчики платформы, разработчики ИТ-сервиса, операторы платформы, поставщики информации и потребители ИТ-сервисов. Инфраструктурные платформы также разделены нами на три типа. Централизованные инструментальные цифровые платформы (тип В) забирают наибольшую долю стоимости трансакции, при этом у данного вида платформ самые высокие затраты на масштабирование. Примерами централизованных инфраструктурных цифровых платформ являются мобильные операционные системы (Apple iOS; Android; SailfishOS; WindowsPhone) и мессенджеры (WhatsApp; Telegram; WeChat; Tamtam; Skape; Avirton; Farechat).

В децентрализованных инфраструктурных цифровых платформах (тип Е) оператор платформы сводит между собой агентов и облегчает трансакции в обмен на небольшую комиссию, при этом актив представляется непосредственно пользователю. Примерами цифровых платформ типа Е являются платформы связанные с арендой жилья (AirBnB; HomeAway; Cian; Hostelworld; Domofond) и платформы в области образования (Edx; Coursera; Udacity; Stepik; Universarium; Eduson). Функциони- 
рование децентрализованных инфраструктурных цифровых платформ направлено на получение бизнес-эффекта от предоставления услуги потребителю, т. е. происходит агрегация отдельных автоматизированных процессов в рамках трансакции.

В гибридных инфраструктурных цифровых платформах типа Н оператор должен тщательно управлять своими отношениями с агентами, поскольку они имеют наименьший контроль, чем при остальных типах платформ. Владение активом децентрализовано, стандарт услуги устанавливается оператором, но ценообразование гибкое, в данном случае хоть и устанавливается владельцем, однако может быть существенно изменено операторами платформы и/или поставщиками информации. Примерами платформ типа Н являются онлайн платежные системы (WePay; PayPal; Amazon Pay; Apple Pay; Robokassa; QIWI).

Главной характеристикой прикладных цифровых платформ является то, что в основе находится бизнес-модель по ведению хозяйственной деятельности. Основной вид деятельности на прикладных цифровых платформах - обмен определенными экономическими ценностями на заданных рынках. Результатом является сделка (обмен) товарами (услугами) между участниками рынка. Основным бенефициаром будет конечный потребитель, решающий бизнес-задачу. Оператор платформы осуществляет регуляторные функции по обеспечению требований законодательства. Централизованная прикладная цифровая платформа (тип С) характеризуется тем, что актив сосредоточен у владельца и он же устанавливает условия, его деятельность заключается в обработке информации о заключении и выполнении сделки между несколькими субъектами экономики. Типичными примерами платформ типа С являются платформы, предоставляющие туристические услуги (Tripadvisor; Skyscanner; Kayak; Aviasales; Tourvisor; Travelata), и платформы, предоставляющие определенные государственные услуги (Gosuslugi.ru; Mos.ru; Gov.uk; Dta.gov.au).

Децентрализованные прикладные цифровые платформы предлагают актив непосредственно пользователю, т. е. всем участникам экономической деятельности - как поставщикам, так и производителям товаров (услуг). Задача платформ типа F - предоставление возможности обмена и облегчения процедуры его реализации за счет повышения прозрачности и упрощения алгоритмизации процесса. Главная задача децентрализованных прикладных цифровых платформ - вовлечение максимально доступного числа участников платформы и максимизация числа трансакций между ними. Типичными примерами платформ типа F являются площадки краундфандинга (Kickstarter; Planeta; IndieGoGo; Starttrack; Boomstarter; Crowdfunder) и платформы по обслуживанию систем купонов и скидок (Groupon; Biglion).

Гибридные прикладные цифровые платформы (тип I) обеспечивают развитие платформенной конкуренции. Среди главных характеристик следует отметить наличие сетевого эффекта (процесс роста клиентской базы является саморазвивающимся) и позитивных обратных связей меду ростом базы потребителей и базы поставщиков. Риск полностью децентрализован, а стандартизация и уровень обслуживания централизованы. Примерами платформ типа I являются платформы поиска работы (Job; Indeed; Upwork; CareerBuilder; Superjob; YouDo) и платформы, связанные с пассажирскими перевозками (Lyft; Uber; Blablacar; City-mobil; Rentmania).

Составленная авторами классификация позволяет применить различные типы цифровых платформ к различным уровням публичного управления. На всех уровнях публичного управления, руководствуясь концепциями Government as a Platform (GaaP) и Government 2.0, необходимо применять цифровые платформы для координации инновационных моделей предоставления публичных услуг и внутреннего взаимодействия между уровнями публичного управления и внутри них. Следует отметить, что цифровые платформы обладают огромным потенциалом для социально-экономического развития общества и получения как социального, так и экономического эффекта. В зависимости от уровня публичного управления нами предложены наиболее целесообразные для применения типы цифровых платформ (рисунок).

Особенно хочется подчеркнуть, что платформ на различных уровнях управления должно быть много, нельзя ограничиваться определенным видом и стандартизировать виды платформ для определенного уровня публичного управления. В сложных системах не существует единственного верного способа управлять, не может быть единой оптимальной стратегии развития. Именно поэтому применение ценностей и принципов agile менеджмента необходимо для создания и использования цифровых платформ в публичном управлении. Постоянное изменение внешней и внутренней среды требует обеспечивать гибкость в применении цифровых платформ, постоянной их модернизации и улучшения качественных характеристик [8]. Стоит измениться условиям, как необходимо изменять и способы управления соответствующими проектами. Предложенная нами 
Цифровые платформы в публичном управлении на основе agile менеджмента

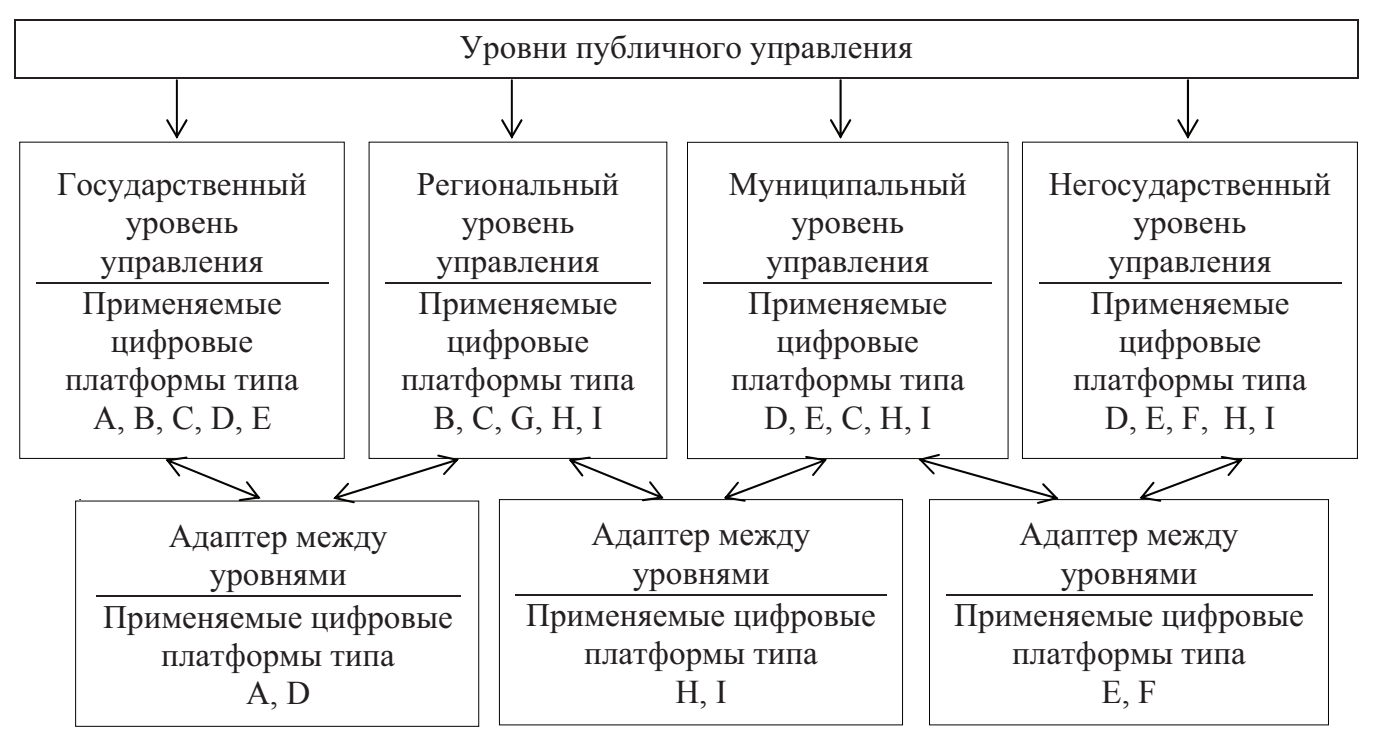

Рисунок. Целесообразные для применения типы цифровых платформ в зависимости от уровня публичного управления и адаптеры между уровнями

классификация отражает только основные ориентиры для внедрения цифровых платформ на различных уровнях публичного управления, в то же время мы не отрицаем, что в применение того или иного типа цифровой платформы будет эффективно в конкрет-

\section{ЛИТЕРАТУРА}

1. Mencken H. L. A New Dictionary of Quotations on Historical Principles from Ancient and Modern Sources / H. L. Mencken. - New York : Encounter Books. - 1942. P. 254 .

2. Brooks. M. Born believers : How our brain creates God / M. Brooks // New Scientist. - Feb 4. - 2009. - URL: htpp://www.Newscientist/article/mg20126941.700-bornbelievers-how-our-brain-creates-god.html

3. Weinberg G. Quality Software Management / G. Weinberg. - New York : Dorset House Pab, 1992. - 325 p.

4. Трещзевский Ю. И. Риск и неопределенность в социально-экономических системах / Ю. И. Трещевский, С. В. Седыкин // Регион : системы, экономика, управление. - 2011. - № 3 (14). - С. 47-50.

5. Озимина Л. А. Цифровое продвижение : теоретические аспекты / Л. А. Озимина, В. А. Плотников //

Среднерусский институт управления-филиал РАНХиГС

Полянин А. В., профессор кафедры «Менеджмент и государственное управление»

E-mail: polyanin.andrei@yandex.ru

тел.: +7 (4862) 71-49-13

Докукина И. А., дочент кафедры «Менеджмент и государственное управление»

E-mail:irentlrs@yandex.ru

тел.: +7 (4862) 71-49-13 ной ситуации и может не совпадать с предложенными нами. В зависимости от типовых характеристик уровня публичного управления нами предложены наиболее целесообразные для применения типы цифровых платформ на данном уровне.

Ученые записки Международного банковского института. - 2019. - № 1 (27). - С. 35-45.

6. Головина T. А. Развитие цифровых платформ как фактор конкурентоспособности современных экономических систем / Т. А. Головина, А. В. Полянин, И. Л. Авдеева // Вестник Пермского университета. Серия : Экономика. - 2019. - Т. 14, № 4. - С. 551-564.

7. Ловчикова Е. И. Цифровая экономика и финансово-инфраструктурное обеспечение : взаимосвязь, проблемы и перспективы / Е. И. Ловчикова, А. И. Солодовник // Банковский сектор : состояние, тенденции и перспективы развития : материалы междунар. науч.-практ. конф., 2018. - С. 103-107.

8. Вертакова Ю. В. Индикаторы оценки цифровой трансформации экономики / Ю. В. Вертакова, М. Г. Клевцова, Ю. С. Положенцева // Экономика и управление. 2018. - № 10 (156). - C. 14-20.

Central Russian Institute of Management, Branch of RANEPA

Polyanin A. V., Professor of the Department of Management and Public Administration

E-mail:polyanin.andrei@yandex.ru

Tel.: + 7 (4862) 71-49-13

Dokukina I. A., Associate Professor of the Department of Management and Public Administration

E-mail: irentlrs@yandex.ru

Tel.: +7 (4862) 71-49-13 